\title{
The service life increasing of bushings by electro-acoustic spraying
}

\author{
Andrei Kochetov ${ }^{1}$, Valentin Minakov ${ }^{1}$, Elena Fisunova ${ }^{1}$, Tatyana Lavrenova, ${ }^{1, *}$, and Luybov \\ Filonenko ${ }^{1}$ \\ ${ }^{1}$ Don State Technical University, Gagarin square, 1, Rostov-on-Don, 344003, Russia
}

\begin{abstract}
The control of obtaining the predicted physico-mechanical properties of protective coatings of machine parts in a complex acoustic field by the method of electroacoustic spraying is carried out through the use of highly concentrated sources of electrical energy. The prediction of the physico-mechanical properties of the hardened surface of machine parts is made on the basis of the experiment planning methodology with nonorthogonal planning matrices. The adequacy of the obtained mathematical models was carried out using the criterion of adequacy of Fisher. Physical fundamentals of mass transfer of materials to a substrate in a complex acoustic field, realized through the transformation of longitudinal vibrations, to longitudinal-torsional, have been developed, while the amplitude of oscillation of the acoustic system is 5 to 15 micrometers. The use of longitudinal-torsional ultrasonic waveguides in this process and ultrafast cooling temperatures make it possible to form elements such as carbides, carbonitrides, and intermetallic compounds on the surface of a conductive material. The subsequent impact with a shift against the surface produced by the waveguide - electrode leads to irreversible microplastic deformations of the surface layer, which provide the specified parameters of surface quality and its physico-mechanical properties.
\end{abstract}

\section{Introduction}

The problem of obtaining predicted physical and mechanical properties and a given service life of multifunctional composite wear-and-heat protective coatings in the form of barrier layers and an increase in the service life of mechanical engineering products and a shaping tool are inherently related. Among all the variety of the latest highly efficient technological processes, a special place here should be given to the method of electroacoustic spraying (ELAS). This method is innovative in the field of multifunctional composite coatings, it allows the formation of nanocrystalline protective films on any conductive materials and substrates [1]. This technology is based on the use of the complex energy of an electric spark and a powerful longitudinal-torsional ultrasonic field.

The aim of this work is to optimize the process of obtaining wear-heat-resistant protective coatings based on the mass transfer of the electrode material and the subsequent formation

\footnotetext{
${ }^{*}$ Corresponding author: bys ka87@mail.ru
} 
of structures with predictable physical and mechanical properties by the method of electroacoustic spraying.

To achieve the above goal, a generalized model of the process of applying wear-heatresistant protective coatings by electro-acoustic spraying was developed, a wide range of studies was carried out, which required the use of modules of the software and hardware complex of the autotechnology control system that were specially developed for these purposes.

\section{Hardware and samples}

The installation of electro-acoustic spraying of multifunctional corrosion-resistant, wearresistant protective coatings of the machine parts is schematically shown in Fig. 1. The block diagram of the electro-acoustic spraying unit consists of: 1- a waveguide with an electrode fixed at its end; 2- part to be hardened; 3- feedback sensor; 4- acoustic system; 5- ultrasonic generator; 6- control system; 7- electronic key; 8- power supply; $\mathrm{C}$ - energy storage.

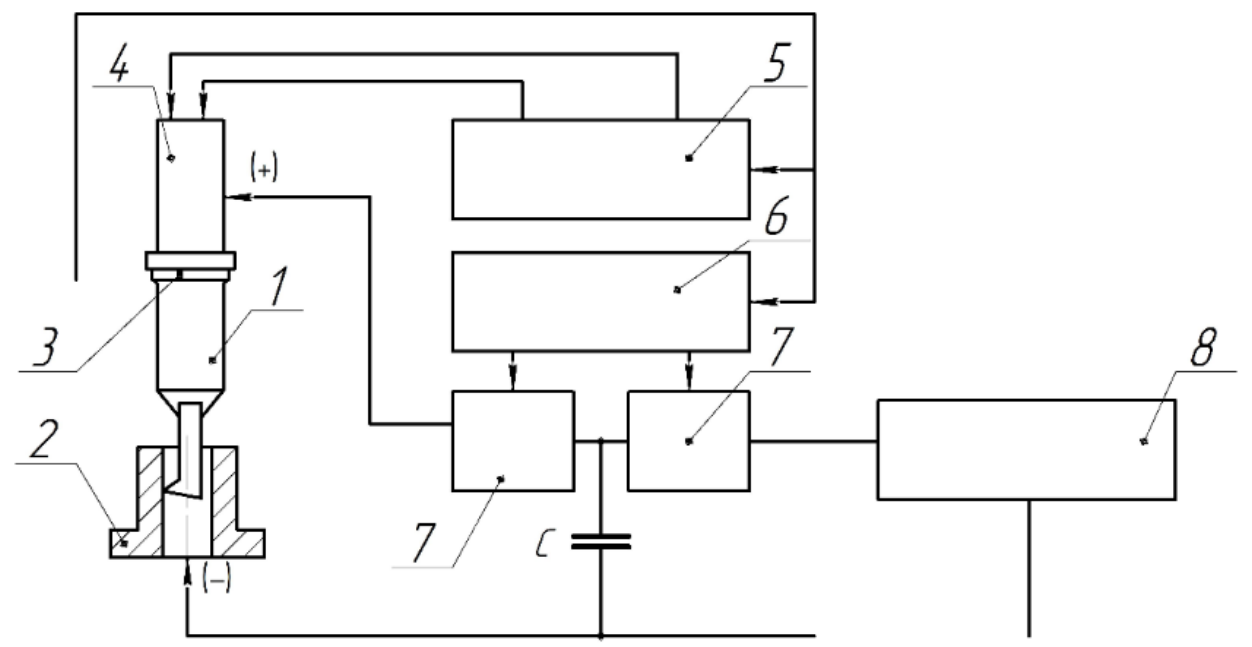

Fig. 1. Block diagram of electro-acoustic spraying.
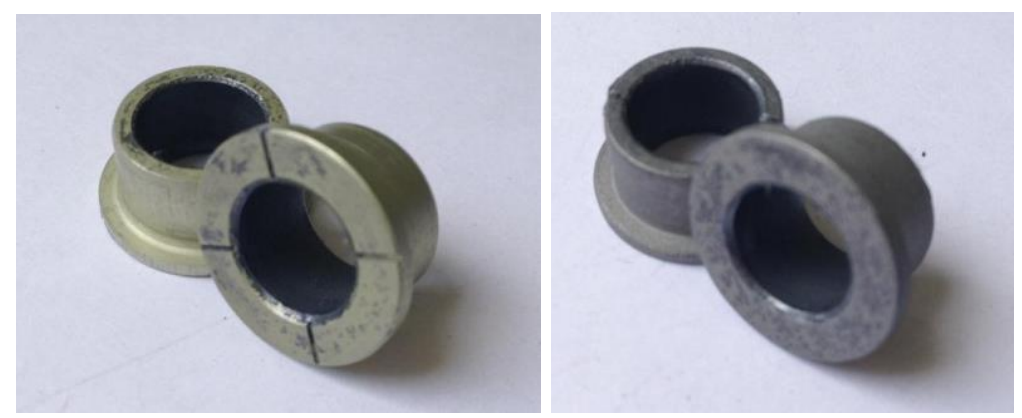

Fig. 2. Reinforced products. 
Optimal shape's selection of the master pulses depending on the magnitude of the erosion of the electrodes.

In most works devoted to the study of the erosion characteristics of metals under the action of pulsed low-voltage discharges in a liquid dielectric medium, the pulse energy and duration are considered as the main parameters of the pulses that determine the erosion characteristics $[2,3]$.

Studies concerning the influence of the shape of the pulse supplied to the spark gap are almost completely absent. Recommendations for choosing the optimal shape of current pulses, given in some works devoted to electrical discharge machining, are given without sufficient experimental and theoretical justification and in some cases are contradictory.

Thus, in the works of B.R. Lazarenko it is indicated that the most advanced are generators that form rectangular current pulses. I.G. Nekrashevich and I.A. Bakuto [4] give an expression for determining the erosion of electrodes under the action of a pulsed discharge, according to which the volume of dispersed metal is

$$
\gamma=\mathrm{A} \frac{1}{t_{P}} \int_{0}^{t_{P}} i^{2} d t
$$

where $A$ - is the coefficient determined by the thermophysical constants of the metal, $t_{P^{-}}$is the pulse duration, $i$ - is the instantaneus current.

Among the works in which the question of choosing the optimal shape of the generator current pulses is discussed should be attributed to the work of Divers [5], in which an attempt is made to develop an electrodynamic hypothesis on the mechanism of erosion proposed by B.R. Lazarenko and N.I. Lazarenko [6].

According to this hypothesis, a dimple on the anode during a pulsed discharge is formed as a result of an instant explosion that occurs when the electron flux is abruptly decelerated by the anode surface, similarly to how an explosion occurs when a body moving at high speed hits a solid obstacle. Divers represents the erosion process in a current pulse of a certain duration as a sequence of instantaneous explosions continuously following each other, the power of which is determined by the instantaneous pulse current. Considering the structural changes in the treated metal surface caused by the thermal effect of explosions, Divers comes to the conclusion that the use of rectangular current pulses is inexpedient. He believes that a decrease in undesirable structural changes should be expected when using current pulses with a gentle current decay at the end of the pulse, for example, using sinusoidal pulses.

It should be noted that this work does not provide an analysis of the action of pulses of various shapes based on any experimental data, and Divers' statements are not convincing.

Consequently, the effect of the pulse shape on the erosion characteristics has hardly been studied. Meanwhile, the solution to this issue is directly related to the definition of pulse parameters that provide optimal processing conditions and to the development of requirements for pulse generators.

Let us consider the physical basis of the process of electrical erosion of an electrode during electro-acoustic spraying. The essence of the process under consideration is that under the action of a voltage applied to the electrode, a breakdown of the air space between the electrode and the treated surface occurs, resulting in a pulsed electric discharge. As a consequence of this phenomenon, electrical erosion of the electrodes occurs. With each single discharge, a very small particle of material is transferred from the electrode to the tool.

The amount of transferred metal mainly depends on the electro-erosive stability of the electrode material and the energy of a single pulse. A number of researchers [7, 8, 9] expressed their views on the mechanism of electrical erosion of metals. However, a sufficiently reasoned theory for the quantitative calculation of the values of the erosional destruction of electrodes has not yet been created. 
Some authors [10] propose to use the energy conservation equation in the following form to calculate the value of electrode erosion:

$$
\left[c_{S}\left(t_{m}-t_{0}\right)+c_{L}\left(t_{b}-t_{m}\right)+c_{V}\left(t_{u}+t_{b}\right)+\lambda+r\right] m=W-W_{\text {nom }}
$$

where $m$ - total value of electrode erosion in g per discharge; $c_{S}, c_{L}, c_{V}$ - average values of specific heat capacities, respectively, of solid, liquid and vapor phases; $t_{0}$ - temperature of electrodes before discharge; $t_{m}, t_{b}, t_{s c h}$ - respectively, the melting and boiling temperatures of the electrode material and the spark channel temperature; $\lambda, r$ - specific heats of melting and evaporation of electrode materials; $W$ - charged capacitor energy; $W_{\text {nom }}$ - the sum of energy losses in the circuit for heating, liquid decomposition, etc.

This equation assumes that all the metal removed from the electrodes during the discharge passes through the vapor phase with the heating of the metal vapor to the temperature of the spark channel $\left(10000^{\circ}\right)$. This assumption is, however, in contradiction with experience. As studies have shown [11], the removal of the metal of the electrodes in a spark discharge occurs in the liquid, vaporous, and sometimes in the solid phase. It is also not true that the temperature of the entire mass of vapors of the substance of the electrodes, which passes into erosion products, can reach $10,000^{\circ} \mathrm{C}$. This temperature actually develops only in the spark channel, which has a very small volume, while the volume of metal vapor generated during the discharge is many times greater than the volume of the spark channel. Equation (2) does not take into account the energy spent on ionization and excitation of vapor atoms, losses for heat removal to electrodes, etc. The processes occurring in the spark gap and on the electrodes during a discharge are taken into account most fully [12] in the following equation:

$$
\begin{array}{r}
c_{L} t_{m} k_{2} \frac{d m}{d t}=W_{S}-\left[k_{1} c_{S}\left(t_{m}-t_{0}\right)+k_{2} \lambda+k_{2} c_{L}\left(t_{b}-t_{m}\right)+k_{4} r\right. \\
\left.+k_{5} c_{V}\left(t_{s c h}-t_{b}\right)+k_{6} W_{e x}+k_{7} W_{i o n}+k_{8} W_{t}^{c}+k_{9} W_{t}^{a}\right]
\end{array}
$$

where $m$ - total value of electrode erosion in $\mathrm{g} ; c_{S}, c_{L}, c_{V}$ - average values of specific heat capacities, respectively, of solid, liquid and vapor phases; $t_{0}$ - temperature of electrodes before discharge; $t_{m}, t_{b}, t_{s c h}$ - respectively, the melting and boiling temperatures of the electrode material and the spark channel temperature; $\lambda, r$ - specific heats of melting and evaporation of electrode materials; $k_{1}, k_{2}, k_{3}, k_{4}, k_{5}$, - coefficients expressing the fraction of the mass of the dispersed material, respectively, heated from $t_{0}$ to $t_{m}$ and from $t$ to $t_{b} ; k_{6}, k_{7}$ - mass fraction coefficients of dispersed metal atoms subjected to excitation and ionization; $k_{8}$ and $k_{9}$ coefficients expressing the fraction of the mass of dispersed metal forming cathode and anode flares; $W_{e x}$ and $W_{i o n}$ - excitation and ionization energy of atoms per unit mass of dispersed metal; $W_{S}$ - energy released in the spark gap of a metal, $W_{t}^{c}$ and $W_{t}^{a}$ - kinetic energy per unit mass of cathode and anode torches.

Calculation of the value of erosion, according to equation (3), requires knowledge of many constants and coefficients, as well as some quantities, some of which are known with more or less confidence, and some are determined by experiments or theoretical calculations [13].

The instantaneous value of the energy transferred to the inter-electrode gap [14] is determined by the expression:

$$
d W_{S}=A_{T} \cdot i^{2} d t
$$

where $A_{T}$ - coefficient determined by the thermophysical constants of the metal.

Thus, the initial data for the study is the mathematical model of the control object, represented by the following second-order differential equation: 


$$
\begin{gathered}
c_{L} t_{m} k_{2} \frac{d^{2} m}{d t^{2}}+\left[k_{1} c_{S}\left(t_{m}-t_{0}\right)+k_{2} \lambda+k_{2} c_{L}\left(t_{b}-t_{m}\right)+k_{4} r+k_{5} c_{V}\left(t_{s c h}-t_{b}\right)\right. \\
\left.+k_{6} W_{e x}+k_{7} W_{i o n}+k_{8} W_{t}^{c}+k_{9} W_{t}^{a}\right] \frac{d m}{d t}=A_{T} i^{2}
\end{gathered}
$$

For convenience of further calculations, we make a change of variables and denote the coefficients in front of the variables as follows

$$
\left\{\begin{array}{c}
T_{1} \dot{x}_{1}=x_{2}-T_{2} x_{1} \\
\dot{x}_{2}=k U
\end{array}\right.
$$

where parameters $T_{1}, T_{2}, k$ - the quantities are constant.

As initial data for numerical calculations, we take [7] the following values of the coefficients $Q_{1}=k_{1} c_{S}\left(t_{m}-t_{0}\right)=0.87 ; Q_{2}=k_{2} \lambda=0.44 ; Q_{3}=k_{3} c_{L}\left(t_{b}-t_{m}\right)=1.97 ;$ $Q_{4}=k_{4} r=6.18 ; Q_{5}=k_{7} W_{\text {ion }}=13.72 ; Q=Q_{1}+Q_{2}+Q_{3}+Q_{4}+Q_{5}=23.18 \mathrm{cal}$; $T_{1}=6836, T_{2}=23.18, k=4.4 ; U_{\max }=0.02 ; t_{f}=60 \mathrm{mks}$.

To find the optimal form of supplying discharge pulses, we formulate the following problem: find a control algorithm $U(t)$ that transfers the object from the position $x_{1}(0)=$ $0, x_{2}(0)=0$ under $t=0$, in a finite time $t_{f}$ into position $x\left(t_{f}\right)=\max x, x^{\prime}\left(t_{f}\right)=0$ with control restrictions $|U|<U_{\text {max }}$.

We will seek a solution to this problem using the Pontryagin maximum principle. We write the functional in the form $J=-x_{1}\left(t_{f}\right) \rightarrow \min$.

The Pontryagin function for the problem under consideration will have the form:

$$
\begin{gathered}
H(\cdot)=\Psi_{1} \frac{\left(x_{2}-T_{2} x_{1}\right)}{T_{1}}+\Psi_{2} k u, \\
\dot{\Psi}_{1}=-\frac{\partial H(\cdot)}{\partial x_{1}}=\frac{T_{2}}{T_{1}} \Psi_{1} \\
\dot{\Psi}_{2}=-\frac{\partial H(\cdot)}{\partial x_{2}}=\frac{\Psi_{1}}{T_{1}}
\end{gathered}
$$

These conditions coincide with the conditions obtained for the previous problem, so it can be written

$$
\begin{gathered}
\Psi_{1}=c_{1} \cdot e^{\frac{T_{2}}{T_{1}} t} \\
\Psi_{2}=c_{2}-\frac{c_{1}}{T_{2}} e^{\frac{T_{2}}{T_{1}} t}
\end{gathered}
$$

Since we are considering a problem with moving ends and a fixed time, it is necessary to take into account the transversality conditions

$$
\frac{\partial B(\cdot)}{\partial x_{1}\left(t_{f}\right)}\left|\frac{x(0)}{x\left(t_{f}\right)}=-\frac{\partial L(\cdot)}{\partial \dot{x}_{1}}\right| t_{f}
$$

where $B(\cdot)=-\Psi_{0} x_{1}\left(t_{f}\right)=x_{1}\left(t_{f}\right)$, and $L(\cdot)$ - lagrangian.

Or 


$$
1=\Psi_{1}\left(t_{f}\right)
$$

The optimal control will be expressed as

$$
u^{*}=\left\{\begin{array}{c}
u_{m} \text { under } 0 \leq t \leq t_{1} \\
-u_{m} \text { under } t_{1}<t \leq t_{f}
\end{array}\right.
$$

Since, by condition, it is required to reach the maximum value $x_{1}$, the optimal control in the first section of the trajectory must be positive. Let's define the control switching point. From the equations of the object we obtain:

$$
\begin{gathered}
x_{2}^{1}=k u_{m} t+c_{3}, \\
x_{2}^{2}=-k u_{m} t+c_{3}, \\
x_{1}^{1}=c_{4} e^{-\frac{T_{2}}{T_{1}} t}+\frac{k u_{m}}{T_{2}} t+\frac{c_{3}}{T_{1}}-\frac{k u_{m}}{T_{2}}, \\
x_{1}^{2}=c_{4} e^{-\frac{T_{2}}{T_{1}} t}+\frac{-k u_{m}}{T_{2}} t+\frac{c_{3}}{T_{1}}-\frac{-k u_{m}}{T_{2}} .
\end{gathered}
$$

Using the initial conditions for the state variables, we obtain

$$
\begin{gathered}
c_{3}^{1}=-k u_{m}, \\
c_{4}^{1}=-\frac{c_{3}^{1} T_{1}}{T_{1}-T_{2}}-k u_{m}+x_{1}^{1},
\end{gathered}
$$

from end conditions

$$
\begin{gathered}
x\left(t_{f}\right)=c_{3}^{2} \cdot e^{-\frac{t_{f}}{T_{1}}}-k u_{m} \\
x\left(t_{f}\right)=c_{4}^{2} e^{-\frac{t_{f}}{T_{2}}}+\frac{c_{3}^{2} T_{1}}{T_{1}-T_{2}} e^{-\frac{t_{f}}{T_{1}}}-k u_{m} .
\end{gathered}
$$

At a moment in time $t_{\text {sch }}$ due to the continuity of the phase trajectory, the equality is done $x_{1}^{1}=x_{1}^{2}$ and $x_{2}^{1}=x_{2}^{2}$, then it can be written

$$
\begin{gathered}
c_{3}^{1} e^{-\frac{t_{s c h}}{T_{1}}}+k u_{m}=c_{3}^{2} e^{\frac{t_{s c h}}{T_{1}}}-k u_{m} \\
c_{4}^{1} e^{-\frac{t_{s c h}}{T_{2}}}+\frac{c_{3}^{1} T_{1}}{T_{1}-T_{2}} e^{-\frac{t_{s c h}}{T_{1}}}+k u_{m}=c_{4}^{2} e^{-\frac{t_{s c h}}{T_{2}}}+\frac{c_{3}^{2} T_{1}}{T_{1}-T_{2}} e^{-\frac{t_{s c h}}{T_{1}}}-k u_{m}
\end{gathered}
$$

The system consisting of six obtained equations (16-21) must be solved with respect to six unknowns: constants, control switching time $t_{s c h}$ and $x_{l}\left(t_{f}\right)$. The following unknown values are obtained in the MathCAD package:

$$
t_{s c h}=0.058 \mathrm{~ms}
$$




$$
\begin{aligned}
& x\left(t_{f}\right)=61.67 \cdot 10^{-3} \mathrm{mg} \\
& c_{3}^{1}=-0.08 \\
& c_{4}^{1}=-0.016 \\
& c_{3}^{2}=1 \\
& c_{4}^{2}=352.387
\end{aligned}
$$

In the Expromt package, we will simulate the behavior of variables $x_{1}, x_{2}$ (Fig. 3) with the obtained optimal control

$$
u^{*}=\left\{\begin{array}{c}
0.002 \text { under } 0 \leq t \leq 0.058 \mathrm{~ms} \\
-0.002 \text { under } 0.058<t \leq 0.06 \mathrm{~ms} .
\end{array}\right.
$$
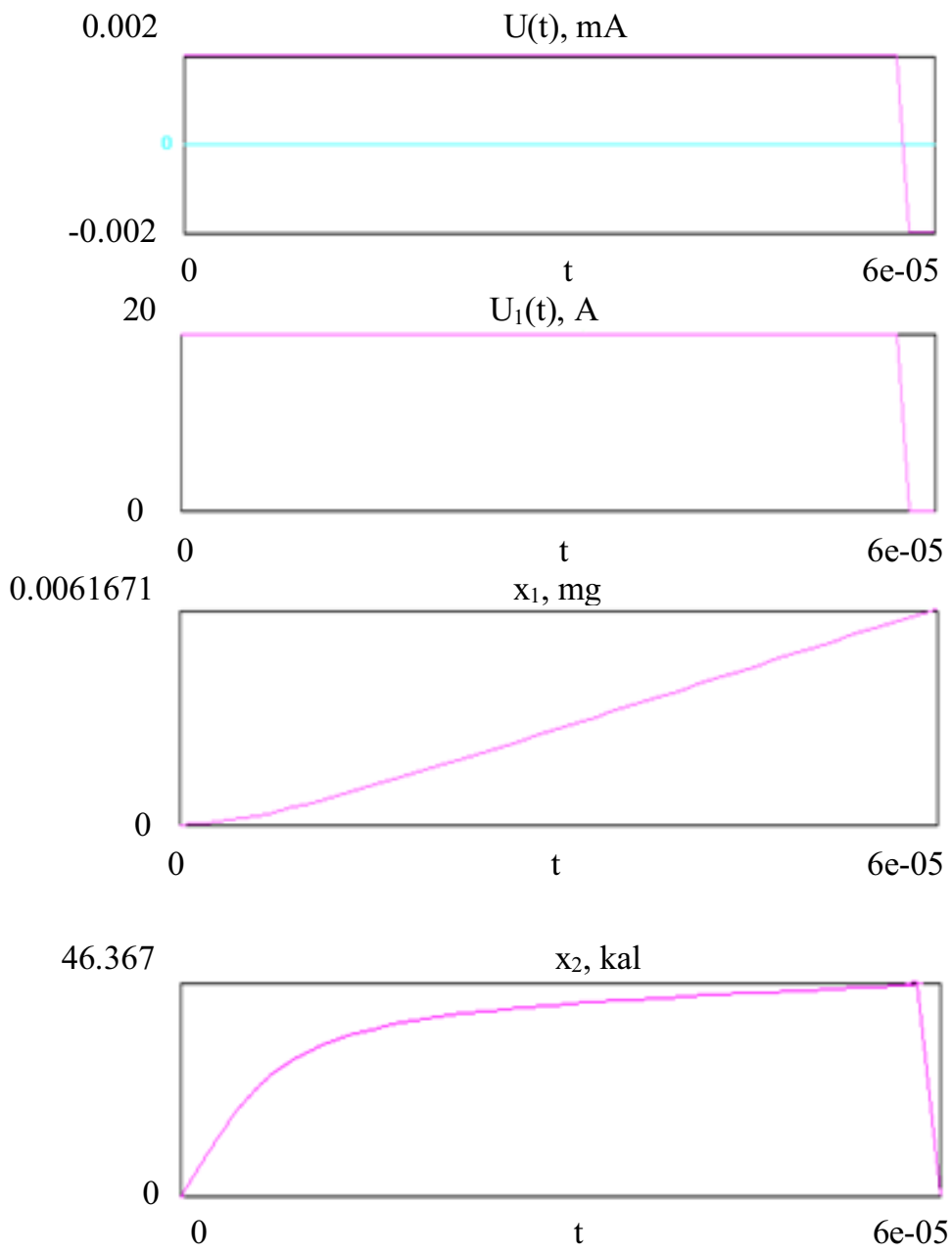

Fig. 3. Transient processes in the system when supplying rectangular pulses with optimal mass transfer. 


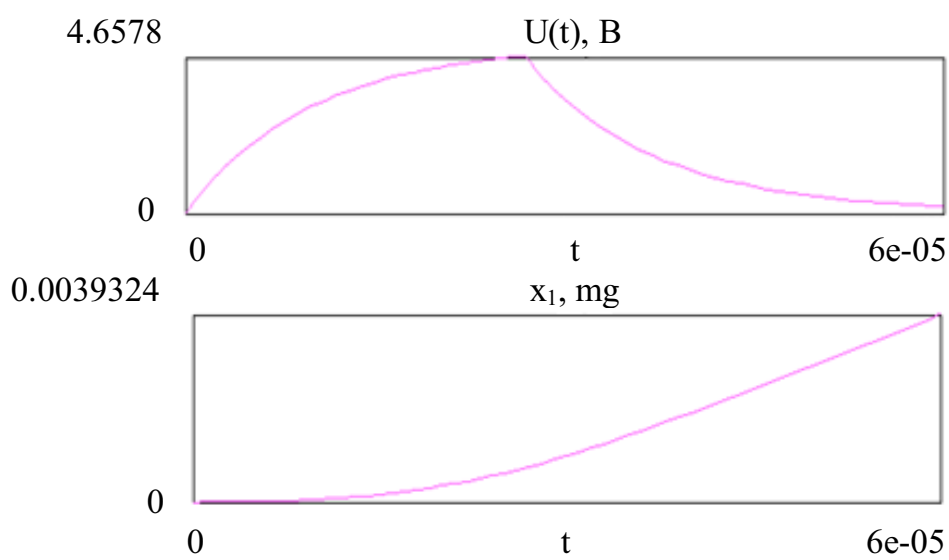

Fig. 4. Transient processes in the ELAS system when supplying discharge pulses using an energy storage device.

\section{Conclusions}

A physical model of electro-acoustic deposition has been obtained, which makes it possible to analyze physical processes and phenomena, as well as the mechanism of formation of a double barrier that prevents the escape of dislocations.

The most acceptable explanation for the hardening of crystals is surface films that prevent dislocations from reaching the surface, i.e. slip suppression.

The ELAS process makes it possible to obtain a "double barrier" that prevents dislocations from reaching the surface. The first barrier is due to microplastic deformation of the surface layer of the hardened product, and the second barrier is due to the sprayed film.

If the shear modulus of the base crystal is less than the shear modulus of the sprayed layer, then the force acting from this layer tends to push the dislocation away from the phase interface, which increases the strength of the product.

The higher the modulus of the film (i.e., the greater), the smaller the number of dislocations in the cluster at the same applied stress. Thus, a crystal covered with a rigid film will plastically deform less than a crystal without a film under the same stress (provided that the average path length of dislocations in the crystal is not very small). This may be the mechanism of the primary hardening of crystals with hard coatings at an early stage of deformation.

- The film changes the shape and magnitude of local stresses caused by the accumulation of dislocations in the base crystal.

- The concentration of stresses caused by the accumulation of dislocations in a crystal covered with a film is less than in a homogeneous one. If the nucleus of the crack caused by the clump grows like a Griffith crack, then the hard-coated material must be more resistant to fracture.

It was found that for a certain ratio the number of edge dislocations in a cluster decreases with an increase in Poisson's ratio. 
A simplified analytical model has been obtained that makes it possible to approximately estimate the behavior of dislocations and the degree of hardening of the material by the ELAS method.

To form the front of the opening and closing of the transistor cascade, as well as to increase the charge dissipation time in order to increase the mass transfer in the interelectrode space with a limited time for supplying the control action, an optimal control law for the key cascade was proposed, which makes it possible to increase the mass transfer by 2 times compared with the classical ELAS method and the charge dissipation time is up to 20 mks, which has a positive effect on the main parameters of the system quality.

The proposed technology for applying anti-corrosion wear-and-heat protective coatings will not only reduce the reduced costs, but also increase the service life of the processed parts and equipment assemblies, which will reduce operating costs by up to $20 \%$. The versatility of the method, the low cost of deposition of multifunctional coatings and the ease of implementation with the possibility of utilizing hard metal waste make this technology economically attractive in comparison with existing methods and technologies.

\section{References}

1. X.F. Zheng, F.X. Qin, H. Wang, Y.-W. Mai, H.X. Peng, Microwave, 151, 62-70 (2017)

2. R. Li, N. Lachman, P. Florin, H.D. Wagner, B.L. Wardle, Composites Science and Technology, 117, 139-145 (2015)

3. B. Yazdani, B. Chen, L. Benedetti, R. Davies, Y. Zhu, Composites Science and Technology, 167, 243-250 (2017)

4. K. Brandt, V. Salikov, H. Özcoban, P. Staron, G.A. Schneider, Composites Science and Technology, 72, 65-71 (2011)

5. W. Li, M. Chen, Z. Zeng, H. Jin, Z. Zhang, Composites Science and Technology, 145, 10-14 (2017)

6. V.V. Ivanov, S.I. Popov, A.V. Kirichek, Key Engineering Materials, 736, 18-22 (2017) doi:10.4028/ www.scientific.net/ KEM.736.18

7. V.V. Ivanov, N.S. Dontsov, A.V. Kirichek, Key Engineering Materials, 736, 105-109 (2017) doi:10.4028/www.scientific.net/ KEM.736.105

8. Lei Guo, Zheng Yan, Xue Dong, Xichun Liu, Fuxing Ye, Composites Science and Technology, 161, 473-482 (2019)

9. X.F. Zheng, F.X. Qin, H. Wang, Y.-W. Mai, H.X. Peng, Inter-fiber stresses in composites with carbon nanotube grafted and coated fibers, 151, 62-70 (2017)

10. S.-J.Joo, M.-H.Yu, W. S. Kim, H.-S. Kim, Composites Science and Technology, 167, 62-70 (2017)

11. M. Sharma, S. Gao, E. Mäder, H. Sharma, J. Bijwe, Composites Science and Technology, 102, 35-50 (2014)

12. B. Jiang, T. Zhang, L. Zhao, Y. Huang, Composites Science and Technology, 140, 3945 (2017)

13. V.V. Ivanov, S.I. Popov, A.V. Kirichek, IOP Conference Series: Materials Science and Engineering, 327(3), 032026 (2018) doi:10.1088/1757-899X/327/3/032026

14. V.V. Ivanov, N.S. Dontsov, A.V. Kirichek, IOP Conference Series: Materials Science and Engineering, 327(3), 032025 (2018) doi:10.1088/1757-899X/327/3/032025 